\title{
Metastatic hepatoid adenocarcinoma of the stomach: a case report and review of the literature
}

Mokrani $\mathrm{A}^{1}$, Behi $\mathrm{K}^{1}$, Sbika $\mathrm{W}^{1} \star$, Mghirbi $\mathrm{F}^{1}$, Yahyoui $\mathrm{Y}^{1}$, Letaief $\mathrm{F}^{1}$, Gabsi $\mathrm{A}^{1}$, Meddeb $\mathrm{K}^{1}$, Ayadi $\mathrm{M}^{1}$, Chraiet $\mathrm{N}^{1}$, Raies $\mathrm{H}^{1}$, Mezlini $\mathrm{A}^{1}$

${ }^{1}$ Department of Medical Oncology- Salah Azaiez Institute, Tunis, University Tunis EL Manar, Tunisia.

\section{Corresponding Author: Wafa SBIKA, MD}

Address: Salah Azaiez Institute, Department of medical oncology, University of Tunis El Manar, Tunisia; E-mail: drwafasbika@gmail.com

Received date: 29 August 2019; Accepted date: 18 September 2019; Published date: 26 September 2019

Citation: Mokrani A, Behi K, Sbika W, Mghirbi F, Yahyoui Y, Letaief F, Gabsi A, Meddeb K, Ayadi M, Chraiet N, Raies H, Mezlini A. Metastatic hepatoid adenocarcinoma of the stomach: a case report and review of the literature. Asp Biomed Clin Case Rep. 2019 Sept 26;2(2):44-47.

Copyright (C) 2019 Mokrani A, Behi K, Sbika W, Mghirbi F, Yahyoui Y, Letaief F, Gabsi A, Meddeb K, Ayadi M, Chraiet N, Raies H, Mezlini A. This is an open-access article distributed under the Creative Commons Attribution License, which permits unrestricted use, distribution, and reproduction in any medium, provided the original work is properly cited.

\section{Abstract}

Hepatoid adenocarcinoma (HAC) is defined as an extrahepatic tumor with hepatocyte differentiation. Hepatoid adenocarcinoma of the stomach (HAS) is a rare type of gastric cancer characterized by unique clinico-pathological features and a poor prognosis. We report a case of a metastatic HAS with a review of the literature.

\section{Keys words}

Hepatoid Adenocarcinoma; Gastric Cancer; Chemotherapy

\section{Background}

Hepatoid adenocarcinoma (HAC) is defined as an extrahepatic tumor with hepatocyte differentiation and may be associated with high plasmatic levels of alpha-feto-protein (AFP). Histology confirms the diagnosis [1].

Hepatoid adenocarcinoma of the stomach (HAS) is a rare type of gastric cancer characterized by unique clinico-pathological features and a poor prognosis. Stomach and esophagus are the most frequent sites but other localizations may be seen such as ovary, uterus, and lungs [2]. In 1970, Bourreille et al. provided the first description of an AFP-producing gastric tumor [3].

Ishikura et al. introduced in 1985 the term of the HAC when reporting seven cases of gastric adenocarcinoma with high serum levels of AFP [1].

Regarding the scarcity of this disease, literature is mostly based on case reports or small case series. Thus, both diagnosis and treatment of this entity remain challenging especially for metastatic HAS.

We report a case of a metastatic HAS with a review of the literature.

\section{Case report}

We present the case of a 32-year-old woman, who complained of abdominal pain. Physical examination found an epigastric hard and painful mass. Abdominal ultrasound revealed many hepatic masses evoking hemangiomas.

Abdominal magnetic resonance imaging showed an 
Citation: Mokrani A, Behi K, Sbika W, Mghirbi F, Yahyoui Y, Letaief F, Gabsi A, Meddeb K, Ayadi M, Chraiet N, Raies H, Mezlini A. Metastatic hepatoid adenocarcinoma of the stomach: a case report and review of the literature. Asp Biomed Clin Case Rep. 2019 Sept 26;2(2):44-47.

Case Report

enlarged liver with many masses and nodules measuring from 1 to $13 \mathrm{~cm}$ consistent with metastases. Gastric endoscopy showed ulcerative antral lesions. A biopsy was performed. The pathological examination concluded to an undifferentiated adenocarcinoma.

However, serum AFP was very high 1500 (normal level <40) leading us to perform a liver biopsy. CEA was under limit of normal. Pathologic examination and revision of the gastric sample showed typical hepatocyte cells. Immunohistochemistry study was negative to $\mathrm{CK}_{7}, \mathrm{CK} 2 \mathrm{O}$, Chromogranin, and Synaptophysin. It finally concluded to a metastatic HAS.
The patient underwent chemotherapy. She received six cycles of XELOX (Capecitabine and Oxaliplatin) with a complete clinical and biological response and radiological partial response estimated to $45 \%$ according to RECIST 1.1 criteria. We opted to maintenance chemotherapy using six cycles of Capecitabine with clinical, biological and radiological stable disease. The systemic treatment was well tolerated. Twenty months after the diagnosis, the patient died of the disease.

\section{Discussion}

HAC is defined as an extrahepatic tumor with hepatocyte differentiation with potentially high levels

\begin{tabular}{|c|c|c|c|c|c|c|c|c|c|}
\hline \multicolumn{10}{|c|}{ Table-1: Reported cases of metastatic HAS } \\
\hline Case & $\begin{array}{l}\text { Author } \\
\text { /year }\end{array}$ & Nationality & $\begin{array}{l}\text { Age } \\
\text { /Sex }\end{array}$ & Metastases & $\begin{array}{c}\text { AFP/ } \\
\text { CEA }\end{array}$ & $\begin{array}{l}\text { Gastric } \\
\text { surgery }\end{array}$ & Chemotherapy & Response & OS \\
\hline 1 & $\begin{array}{c}\text { Shima } \\
\mathrm{da} / 200 \\
2\end{array}$ & Japanese & $71 / \mathrm{F}$ & Liver & $\begin{array}{c}5190 / \\
\text { NA }\end{array}$ & No & $\begin{array}{c}\text { Cisplatin/Paclita } \\
\text { xel }\end{array}$ & $\begin{array}{l}\text { Complete } \\
\text { response }\end{array}$ & - \\
\hline 2 & $\begin{array}{c}\text { Shima } \\
\mathrm{da} / 200 \\
2\end{array}$ & Japanese & $\begin{array}{c}63 / \\
M\end{array}$ & Liver & $\begin{array}{l}156 / \\
\text { NA }\end{array}$ & No & $\begin{array}{l}\text { Weekly } \\
\text { paclitaxel }\end{array}$ & $\begin{array}{l}\text { Complete } \\
\text { response }\end{array}$ & - \\
\hline 3 & $\begin{array}{l}\text { Chiba/ } \\
2005\end{array}$ & Japanese & $\begin{array}{c}47 / \\
M\end{array}$ & Liver & $\begin{array}{l}606.8 \\
\text { /ULN }\end{array}$ & Yes & $\begin{array}{l}\text { 5FU/Cisplatin/Et } \\
\text { oposide }\end{array}$ & $\begin{array}{c}\text { Partial } \\
\text { response }\end{array}$ & $\begin{array}{c}14 \\
\text { month } \\
\mathrm{s}\end{array}$ \\
\hline 4 & $\begin{array}{c}\text { Takaye } \\
\text { ma/20 } \\
07\end{array}$ & Japanese & $\begin{array}{c}64 / \\
M\end{array}$ & Liver & $\begin{array}{l}1497 . \\
8 / 727\end{array}$ & No & $\begin{array}{c}\text { Doxorubicin/My } \\
\text { tomicin/5FU }\end{array}$ & stable & $\begin{array}{c}9 \\
\text { month } \\
\mathrm{s}\end{array}$ \\
\hline 5 & $\begin{array}{c}\text { Takaha } \\
\text { shi/20 } \\
09\end{array}$ & Japanese & $\begin{array}{c}51 / \\
M\end{array}$ & Liver & $\begin{array}{c}91 / N \\
A\end{array}$ & No & $\begin{array}{c}\text { Cisplatin/Capeci } \\
\text { tabine }\end{array}$ & $\begin{array}{l}\text { Complete } \\
\text { response }\end{array}$ & $\begin{array}{c}7 \\
\text { years }\end{array}$ \\
\hline 6 & $\begin{array}{l}\operatorname{Lin} / 20 \\
09\end{array}$ & Chinese & $56 / F$ & Liver & $\begin{array}{c}9457 / \\
\text { NA }\end{array}$ & Yes & $\begin{array}{c}\text { FOLFIRI/Bevaciz } \\
\text { umab }\end{array}$ & stable & $\begin{array}{c}20 \\
\text { month } \\
\mathrm{s}\end{array}$ \\
\hline 7 & $\begin{array}{c}\text { Galvez } \\
- \\
\text { Mulno } \\
\text { z/2009 }\end{array}$ & European & $\begin{array}{l}75 / \\
M\end{array}$ & Liver/Nodes & $\begin{array}{c}4500 / \\
460\end{array}$ & Yes & Sorafenib & Progression & $\begin{array}{c}8 \\
\text { month } \\
\mathrm{s}\end{array}$ \\
\hline 8 & $\begin{array}{c}Y e / 201 \\
3\end{array}$ & Chinese & $\begin{array}{l}54 / \\
M\end{array}$ & Lung & $\begin{array}{c}99 / \mathrm{UL} \\
\mathrm{N}\end{array}$ & Yes & $\begin{array}{c}\text { Cisplatin/Capeci } \\
\text { tabine }\end{array}$ & $\begin{array}{l}\text { Complete } \\
\text { response }\end{array}$ & $\begin{array}{c}20 \\
\text { month } \\
\mathrm{s}\end{array}$ \\
\hline 9 & $\begin{array}{c}\text { Ye/201 } \\
3\end{array}$ & Chinese & $61 / F$ & Spleen & $\begin{array}{c}>500 \\
00 / U L \\
N\end{array}$ & No & Gemcitabine & Progression & $\begin{array}{c}18 \\
\text { month } \\
\mathrm{s}\end{array}$ \\
\hline 10 & $\begin{array}{c}\text { Ahn/2 } \\
013\end{array}$ & Korean & $\begin{array}{c}68 / \\
M\end{array}$ & Liver & $\begin{array}{c}\mathrm{NA} / \mathrm{N} \\
\mathrm{A}\end{array}$ & Yes & Cisplatin S-1 & $\begin{array}{l}\text { Complete } \\
\text { response }\end{array}$ & $\begin{array}{c}9 \\
\text { years }\end{array}$ \\
\hline 11 & $\begin{array}{c}\text { Nagai/ } \\
2014\end{array}$ & Japanese & $\begin{array}{c}62 / \\
M\end{array}$ & Liver & $\begin{array}{c}\mathrm{NA} / \mathrm{N} \\
\mathrm{A}\end{array}$ & Yes & $\begin{array}{l}\text { Pacltaxel/Carbo } \\
\text { platin/Sorafenib }\end{array}$ & $\begin{array}{l}\text { Partial } \\
\text { response }\end{array}$ & $\begin{array}{c}2 \\
\text { years }\end{array}$ \\
\hline
\end{tabular}


Citation: Mokrani A, Behi K, Sbika W, Mghirbi F, Yahyoui Y, Letaief F, Gabsi A, Meddeb K, Ayadi M, Chraiet N, Raies $\mathrm{H}$, Mezlini A. Metastatic hepatoid adenocarcinoma of the stomach: a case report and review of the literature. Asp Biomed Clin Case Rep. 2019 Sept 26;2(2):44-47.

Case Report

\begin{tabular}{|c|c|c|c|c|c|c|c|c|c|}
\hline 12 & $\begin{array}{c}\text { Simmet/ } \\
2017\end{array}$ & European & $\begin{array}{l}64 / \\
M\end{array}$ & Liver & $\begin{array}{l}2600 / \\
\text { ULN }\end{array}$ & No & $\begin{array}{c}\text { Cisplatin/Etopos } \\
\text { ide }\end{array}$ & $\begin{array}{l}\text { Complete } \\
\text { response }\end{array}$ & $\begin{array}{c}9 \\
\text { years }\end{array}$ \\
\hline 13 & $\begin{array}{c}\text { Simmet/ } \\
2017\end{array}$ & European & $60 / F$ & Liver & $\begin{array}{l}6700 \\
0 / 176\end{array}$ & No & $\begin{array}{c}\text { Cisplatin/Etopos } \\
\text { ide }\end{array}$ & Progression & $\begin{array}{c}23 \\
\text { month } \\
\mathrm{s}\end{array}$ \\
\hline & & & & & & & $\begin{array}{l}\text { Capecitabine/Ox } \\
\text { aliplatin }\end{array}$ & \multirow[b]{2}{*}{$\begin{array}{l}\text { Partial } \\
\text { response }\end{array}$} & \multirow{2}{*}{$\begin{array}{c}20 \\
\text { month } \\
\mathrm{s}\end{array}$} \\
\hline 14 & $\begin{array}{c}\text { Mokrani } \\
/ 2018\end{array}$ & Tunisian & $33 / F$ & Liver & $\begin{array}{l}1500 / \\
\text { ULN }\end{array}$ & No & $\begin{array}{l}\text { Maintenance by } \\
\text { Capecitabine }\end{array}$ & & \\
\hline
\end{tabular}

of alpha-feto-protein (AFP) [4]. Pathological examination confirms the diagnosis when it founds typical hepatocyte cells and detects by immunohistochemical study an overexpression of AFP [4].

HAS is a rare subtype of gastric tumors characterized by hepatoid differentiation and high serum levels of AFP [3]. The pathogenesis is still unclear. It may be due to a cellular transdifferentiation from glandular to hepatoid type [4].

The implication of $\mathrm{H}$. Pylori infection in this subtype is not established. Patients are usually adults, aged from 44 to 87 years. There is a male predominance with a sex ratio about 2.3. Most frequent symptoms are unspecific including epigastric pain and asthenia [5].

To our knowledge, only fourteen cases of metastatic hepatoid gastric carcinoma were described in the literature including our patient. The table below (Table-1) summarizes information on these cases [6$14]$.

Most frequent sites of metastases are nodes and the liver. In our case, the patient presented with voluminous hepatic metastases and a high serum level of AFP.

Curative surgery when early detection is possible may be associated with healing. Treatment of localized tumors consists of radical surgery when feasible followed by adjuvant chemotherapy including $5 \mathrm{FU}$, leucovorin, Cisplatin and Epirubicin [5].

EGFR, KRAS, and BRAF mutations were frequently reported as well as overexpression of HER, which implies the possible use of targeted therapies in the metastatic setting [15].

In our case, those mutations have not been assessed. Compared to gastric carcinomas with no hepatoid differentiation, the prognosis is worse with a global 5-year-survival rate of $9 \%[5]$.

\section{Conclusion}

HAS is a rare and aggressive subtype of gastric tumors characterized by a hepatoid differentiation and potentially increased AFP serum levels. Management of metastatic disease is controversial. We reported the case a metastatic HAS treated by XELOX.

Additional systemic treatments are yet to be explored to overcome the poor global prognosis. In this context, targeted therapies represent an interesting alternative especially due to the many molecular mutations.

\section{References}

[1] Ishikura H, Ishiguro T, Enatsu C, Fujii H, Kakuta Y, Kanda M, Yoshiki T. Hepatoid adenocarcinoma of the renal pelvis producing alpha-fetoprotein of hepatic type and bile pigment. Cancer. 1991 Jun 15;67(12):3051-56. [PMID: 1710536]

[2] Fornasa F. Soft-Tissue Localization of Hepatoid Adenocarcinoma: First Case Report. Case Rep Oncol. 2010 Jul 3;3(2):212-17. [PMID: 20740199] 
[3] Bourreille J, Metayer P, Sauger F, Matray F, Fondimare A. Existence of alpha feto protein during gastric-origin secondary cancer of the liver. Presse Med. 1970 Jun 6;78(28):1277-78. [PMID: 5426134]

[4] Simmet V, Noblecourt M, Lizée T, Morvant B, Girault S, Soulié P, Capitain O. Chemotherapy of metastatic hepatoid adenocarcinoma: Literature review and two case reports with cisplatin etoposide. Oncol Lett. 2018 Jan;15(1):48-54. [PMID: 29387209]

[5] Inagawa S, Shimazaki J, Hori M, Yoshimi F, Adachi S, Kawamoto T, Fukao K, Itabashi M. Hepatoid adenocarcinoma of the stomach. Gastric Cancer. 2001;4(1):43-52. [PMID: 11706627]

[6] Shimada S, Hayashi N, Marutsuka T, Baba Y, Yokoyama S, Iyama K, Ogawa M. Irinotecan plus lowdose cisplatin for alpha-fetoprotein-producing gastric carcinoma with multiple liver metastases: report of two cases. Surg Today. 2002;32(12):1075-80. [PMID: 12541026]

[7] Chiba N, Yoshioka T, Sakayori M, Mikami Y, Miyazaki S, Akiyama S, Otsuka K, Yamaura G, Shibata H, Kato S, Kato S, Ishioka C. AFP-producing hepatoid adenocarcinoma in association with Barrett's esophagus with multiple liver metastasis responding to paclitaxel/CDDP: a case report. Anticancer Res. 2005 Jul-Aug;25(4):2965-68. [PMID: 16080552]

[8] Takeyama H, Sawai H, Wakasugi T, Takahashi H, Matsuo Y, Ochi N, Yasuda A, Sato M, Okada Y, Funahashi H, Akamo Y, Manabe T. Successful paclitaxel-based chemotherapy for an alphafetoprotein-producing gastric cancer patient with multiple liver metastases. World J Surg Oncol. 2007 Jul 16;5:79. [PMID: 17634124]

[9] Takahashi T, Kochi M, Kanamori N, Kaiga T, Funada T, Fujii M, Takayama T. Complete remission with FLEP chemotherapy for multiple liver metastasis from alpha-fetoprotein-producing gastric cancer-report of a case. Gan To Kagaku Ryoho. 2009 Nov;36(11):1885-88. [PMID: 19920393]

[10] Lin CW, Hsu CC, Chang HC, Sun YC, Sun PL, Hsu CY, Perng DS. Hepatoid adenocarcinoma of the stomach with liver metastasis mimicking hepatocellular carcinoma: a case report. Cases J. 2009 Aug 11;2:6317. [PMID: 19918575]

[11] Gálvez-Muñoz E, Gallego-Plazas J, GonzalezOrozco V, Menarguez-Pina F, Ruiz-Maciá JA, Morcillo
MA. Hepatoid adenocarcinoma of the stomach - a different histology for not so different gastric adenocarcinoma: a case report. Int Semin Surg Oncol. 2009 Aug 12;6:13. [PMID: 19674468]

[12] Ye MF, Tao F, Liu F, Sun AJ. Hepatoid adenocarcinoma of the stomach: a report of three cases. World J Gastroenterol. 2013 Jul 21;19(27):443742. [PMID: 23885160]

[13] Ahn JS, Jeon JR, Yoo HS, Park TK, Park CK, Sinn DH, Paik SW. Hepatoid adenocarcinoma of the stomach: an unusual case of elevated alphafetoprotein with prior treatment for hepatocellular carcinoma. Clin Mol Hepatol. 2013 Jun;19(2):173-78. [PMID: 23837142]

[14] Nagai Y, Kato T, Harano M, Satoh D, Choda Y, Tokumoto N, Kanazawa T, Matsukawa H, Ojima Y, Idani H, Shiozaki S, Okajima M, Ninomiya M, Ohno S. A case of AFP-producing esophagogastric junction cancer with liver metastases with a good response to chemotherapy. Gan To Kagaku Ryoho. 2014 Nov;41(12):2349-51. [PMID: 25731519]

[15] Chun H, Kwon SJ. Clinicopathological characteristics of alpha-fetoprotein-producing gastric cancer. J Gastric Cancer. 2011 Mar;11(1):23-30. [PMID: 22076198] 